\title{
High Long-term Survival of Bulk Femoral Head Autograft for Acetabular Reconstruction in Cementless THA for Developmental Hip Dysplasia
}

\author{
Mitsunari Kim MD, Toru Kadowaki MD
}

Received: 25 February 2009/ Accepted: 22 February 2010/Published online: 23 March 2010

(C) The Author(s) 2010. This article is published with open access at Springerlink.com

\begin{abstract}
Background Deficient acetabula associated with acetabular dysplasia cause difficulty achieving adequate coverage of the acetabular component during THA. Autografting with the removed femoral head has been used for several decades to achieve better coverage, but the long-term benefits of this technique remain controversial, with some series reporting high rates of graft resorption and collapse. Questions/purposes We evaluated the fate of bulk femoral head autograft for acetabular reconstruction in cementless THA for developmental hip dysplasia.

Patients and Methods We retrospectively reviewed 70 patients (83 hips) (68 women, two men) with a mean age of 57 years at index surgery. According to the classification of Crowe et al. for hip dysplasia, 10 hips were classified as Type I, 45 as Type II, 19 as Type III, and nine as Type IV. Minimum followup was 9 years (mean, 11 years; range, 914 years).

Results We observed no collapsed grafts. In all patients we observed disappearance of the host-graft interface and appearance of radiodense bands in the grafts bridging host iliac bone and at the lateral edges of the acetabular sockets; remodeling with definite trabecular reorientation was seen

Each author certifies that he or she has no commercial associations (eg, consultancies, stock ownership, equity interest, patent/licensing arrangements, etc) that might pose a conflict of interest in connection with the submitted article.

Each author certifies that his or her institution approved the human protocol for this investigation, that all investigations were conducted in conformity with ethical principles of research, and that informed consent for participation in the study was obtained.
\end{abstract}

M. Kim ( $ه)$, T. Kadowaki

Department of Orthopedics, Suita Municipal Hospital, 13-20,

2 Choume, Katayamachou, Suita City, Osaka, Japan

e-mail: gskim@iris.eonet.ne.jp in $90 \%$. The 10-year survival rate without acetabular revision for any reason was 94\%. The mean Merle d'Aubigné and Postel hip score improved from a mean of 9.1 preoperatively to 17.2 at last followup.

Conclusions Cementless THA combined with autologous femoral bone graft in patients with developmental dysplasia resulted in a high rate of survival. Structural bone grafting achieved a stable construct until osseointegration occurred. We believe the radiodense bands represent a radiographic sign of successful completion of repair of the deficient acetabulum. Congruous and stable contact of the cancellous portion of the graft to the host bed by impaction and use of improved porous cementless sockets may be associated with successful socket survival.

Level of Evidence Level IV, therapeutic study. See Guidelines for Authors for a complete description of levels of evidence.

\section{Introduction}

When THA is required in a patient with developmental dysplasia of the hip (DDH), bone deficiency in the acetabular roof often precludes conventional methods of socket implantation. Options for obtaining adequate bone coverage for stable fixation of the acetabular component have included use of a small cup with medial or high anatomic positioning of the socket [19, 23]. Augmented bone graft prepared from resected femoral head to the defective acetabulum is another option [2, 6, 7, 24-27, 29]. Bulk bone grafting offers advantages: the cup may be placed in an anatomic position rather than a high one, it provides support for the acetabular component, and if incorporated it would provide beneficial bone stock for any 
future revision surgery. Although the importance of coverage and initial stability of the socket is accepted as a principle, numerous authors have reported high rates of autograft resorption, collapse of the bone graft, and socket loosening [7, 8, 10, 12, 21, 22, 28, 31]. Mulroy and Harris [22] reported failure of $46 \%$ of their cemented series by 11.8 years. In comparison to the failure rate reported by Mulroy and Harris [22], survival of $94 \%$ at 6.6 years was reported by Morsi et al. [21] although they also reported mild or moderate resorption of $69 \%$ of their 16 cementless series. Spangehl et al. reported a survival rate of $91 \%$ at 10 years using a cementless acetabular component [31].

Numerous studies have examined the use of uncemented THA with femoral head autografts to augment socket coverage with bone $[1,9,10,13,21,28,30,31]$. Shetty et al. [28] described 15 hips with acetabular dysplasia treated with uncemented THA in conjunction with bulk autografts from femoral heads with no cases of loosening or revision at 8 to 11 years. Ito et al. [13] described 15 dysplastic hips treated with uncemented cups combined with femoral head bone grafting and reported six of the 15 cups had a mean vertical migration of $4.5 \mathrm{~mm}$ within 5 years after surgery. In that study, however, serial radiographs did not show any progression of migration or rotation of the socket after 5 years at least up to a mean followup of 10.6 years. Hendrich and Mehling [9] reported 55 uncemented cups with bone grafting in patients followed a mean of 10 years and substantial socket migration was observed in 19 hips. In some of these studies, the authors obtained initial mechanical strength by using the eburnated superior portion of femoral head [28] or a portion of femoral head that includes the calcar [12]. We believe the variations in survival suggest technique may be important in the survival of the cups. Hemispheric porous sockets and greater surface contact between bone grafts and living host bone should reduce the early interfacial stresses on the graft and stresses between the graft and host bed and thus enhance the chance of survival. There also may be differing rates of survival with cemented and uncemented sockets placed on bulk autografts.

Using particular techniques to implant the graft and socket, we therefore determined (1) the 10-year survival rate with loosening, (2) coverage of the acetabular component by host bone, (3) chronologic radiographic changes in grafted bone during repair of acetabular bone deficiency in dysplastic hips, (4) leg lengthening achieved by the autografts, and (5) functional outcomes.

\section{Patients and Methods}

We retrospectively reviewed 70 patients (68 women, two men) (83 dysplastic hips) treated by THA using
Table 1. Demographic characteristics

\begin{tabular}{ll}
\hline Characteristic & Value \\
\hline Age (years)* & $57(33-72)$ \\
Height $(\mathrm{cm})^{*}$ & $152(137-165)$ \\
Weight (kg)* & $54(36-78)$ \\
Gender (male/female) & $2 / 68$ \\
Crowe type (number of hips) & \\
I & 10 \\
II & 45 \\
III & 19 \\
IV & 9 \\
\hline
\end{tabular}

* Values are expressed as means, with ranges in parentheses.

noncemented socket fixation to the original acetabulum and acetabular defects reconstructed by grafting bulk bone from femoral heads between January 1994 and November 1998. The mean age of the patients at the time of index arthroplasty was 57 years (range, 33-72 years). The mean height of the patients was $152 \mathrm{~cm}$ (range, $137-165 \mathrm{~cm}$ ) and their mean body weight was $54 \mathrm{~kg}$ (range, $36-78 \mathrm{~kg}$ ) (Table 1). The degrees of acetabular dysplasia as classified according to the criteria described by Crowe et al. [4] were Type I in 10 hips (12\%), Type II in 45 (54\%), Type III in $19(23 \%)$, and Type IV in nine (11\%). Nine hips had been treated previously with one or more surgical procedures. Five patients (six hips) were lost to followup. Three patients (three hips) were lost to followup owing to death before followup had reached 9 years. Two patients (three hips) were lost to followup owing to loss of contact. These six hips had well-fixed acetabular components at the last visits (12 to 36 months postoperatively). Thus, 65 patients (77 hips) were available for this study. The minimum followup was 9 years (mean, 11 years; range, 9-14 years). We obtained prior approval for the study from the Institutional Review Board of Suita Hospital and consent from all patients was obtained before the operations.

All surgical procedures were performed by the senior author (TK). A posterolateral approach was used for 74 hips, with a transtrochanteric approach for the remaining three hips. In 39 hips, the uncemented titanium porous acetabular component was fixed to the pelvis with screws. In the remaining 38 hips, initial stability was achieved using a tight press-fit technique without additional screws for socket fixation. Superior segmental deficiencies of the acetabular bone were augmented with bulk autograft prepared from the ipsilateral femoral head. The grafting was accomplished with the following four steps: (1) The lateral cancellous portion of the bulk bone graft was shaped with a reciprocating bone saw (Conmed Linvatec, Largo, FL) to match the convexity of the pseudoacetabulum for accurate contact to the pseudoacetabular floor (Fig. 1). (2) The 


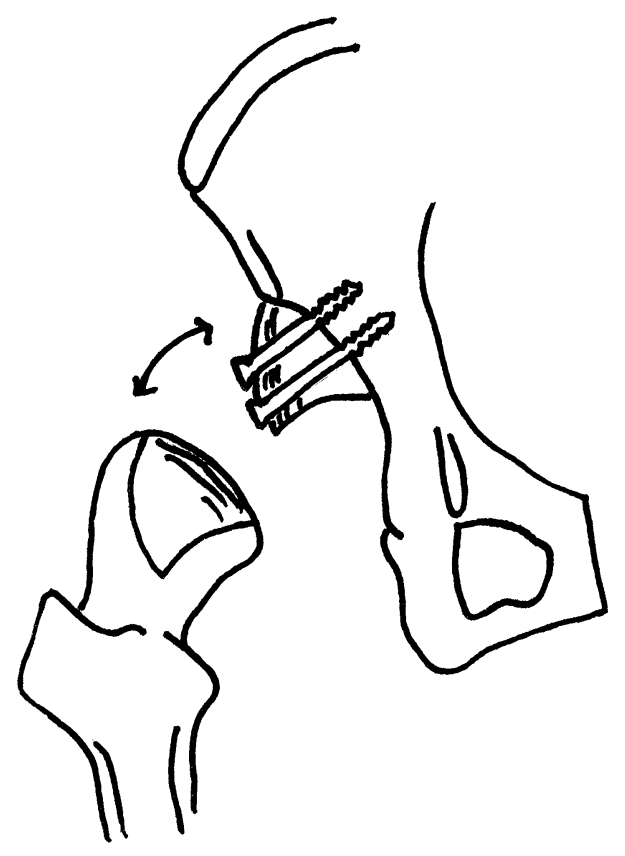

Fig. 1 A diagram illustrates the operative method for bone grafting. The subchondral portion of the femoral head was faced laterally while the cancellous interior of the femoral head was shaped to fit against the pseudoacetabulum.

pseudoacetabular floor was reamed to expose cancellous bone and multiple drill holes were made to facilitate invasion of fibrovascular repair tissue from the iliac bone marrow into the grafted bone (creeping substitution). (3) The cancellous surface of the graft was impacted in the prepared bed using a hammer to ensure close contact of the graft to the graft bed. (4) The graft was fixed securely to the host with screws penetrating outer and inner walls of the iliac bone to prevent micromotion between graft and host bone and to facilitate growth of repairing connective tissue into the graft. For fixation of graft bone, titanium screws (Stryker Orthopaedics, Mahwah, NJ) or absorbable radiolucent polylactide screws (Takiron, Osaka, Japan) [3] were used in 10 hips and 67 hips, respectively. An Osteonics ${ }^{\circledR}$ Dual Geometry cup (Stryker Orthopaedics) was used in 27 hips, and a PSL ${ }^{\circledR}$ cup (Stryker Orthopaedics) was used in 50 hips. The median cup diameter was $48 \mathrm{~mm}$ (range, 42 $52 \mathrm{~mm}$ ). The diameter of the prosthetic femoral head was $26 \mathrm{~mm}$ in all hips. Fixation of the femoral stem was without cement in 70 hips and with cement in seven hips.

All patients were allowed to stand and walk with two crutches and nonweightbearing within 2 days after surgery. All received ROM exercise by physiotherapy until 3 weeks, and began walking exercises with partial weightbearing $(50 \%$ of normal weightbearing) with crutches during 3 to 6 weeks, followed by gradual return to full weightbearing. Patients were permitted to return to work 2 months postoperatively.

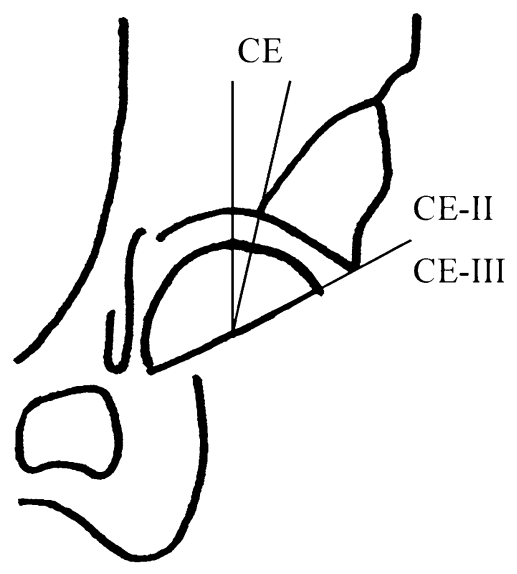

Fig. 2 A diagram illustrates the method of evaluating graft coverage. $\mathrm{CE}=$ angle between the vertical line of the socket center and the original lateral edge of the acetabulum (which was also the medial edge of the graft bone) immediately postoperatively; CE II = angle between the vertical line of the socket center and the lateral edge of the graft bone immediately postoperatively; $\mathrm{CE}$ III = angle between the vertical line of the socket center and the lateral edge of the graft bone on the last visit.

We followed patients at 2, 4, 6, 9, and 12 months after surgery, and every 6 or 12 months thereafter. We obtained the Merle d'Aubigné and Postel hip score [20] at 12 months and yearly thereafter. Measurement of leg length was evaluated by comparing the position of the midportion of the lesser trochanter to the interteardrop line on preoperative and postoperative radiographs. We defined a high hip center as one with the center greater than $35 \mathrm{~mm}$ proximal to the interteardrop line.

Standard AP radiographs were taken immediately after the operation; 2, 4, 6, 9, and 12 months after the operation; and every 6 or 12 months thereafter. All assessments were made by one of us (MK). The degree of coverage (Fig. 2) or percentage of horizontal coverage by host and graft bone (Fig. 3) was determined on postoperative radiographs [32]. As a measure of coverage, we calculated three center-edge (CE) angles: (1) CE, the angle between the vertical line of the socket center and the original lateral edge of the acetabulum (which was also the medial edge of the graft bone) immediately postoperatively; CE II, the angle between the vertical line of the socket center and the lateral edge of the graft bone immediately postoperatively; and CE III, the angle between the vertical line of the socket center and the lateral edge of the graft bone at the last visit (Fig. 2). The projected percentage horizontal coverage above the socket was determined using the following formula: (horizontal host bone distance/horizontal distance between the medial and the lateral edge of the socket) $\times 100 \%$ (Fig. 3). On final followup radiographs, the horizontal host bone distance represented the distance of the graft and host bone because the interface between the host bone and graft bone was obscure. 


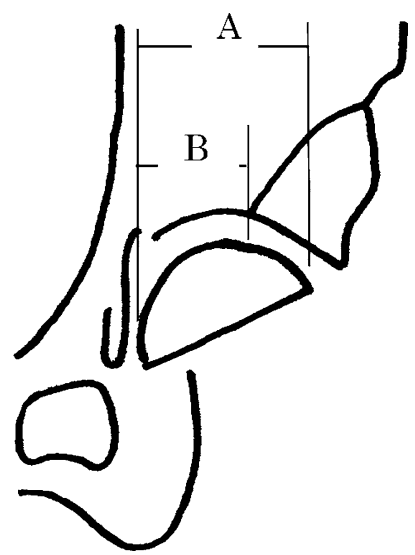

Fig. 3 A diagram illustrates the projected percentage horizontal coverage above the socket calculated as (horizontal host bone distance $[\mathrm{B}] /$ horizontal distance between the medial and the lateral edge of the socket $[\mathrm{A}]) \times 100 \%$.

Incorporation and remodeling of grafted bone were analyzed according to the methods described by Knight et al. [15]. Bridging trabeculation across the graft-host interface was judged to be present when the interface apparent on early radiographs could no longer be seen distinctly. Rounding off of the protruding edge of the graft beyond the cup or a change in graft density in areas where the graft was not stressed was considered an indicator of revascularization. Reorientation of the trabecular pattern in the graft to match the normal trabecular orientation of the acetabular dome also was noted. Vertical and horizontal positions of the acetabular socket were determined according to the methods described by Russotti and Harris [27]. Radiolucent lines at the acetabular bone-implant interface were recorded as described by DeLee and Charnley [5]. Inclination angle of the socket was determined as the angle subtended by the face of the socket and the interteardrop line on AP radiographs. Probable or definite acetabular loosening was defined as a change in socket position, socket migration, screw fracture, or complete radiolucency at the bone-implant interface of $2 \mathrm{~mm}$ or greater in any zone. Linear head penetration into the polyethylene liner was measured using the techniques described by Livermore et al. [17].

Incorporation and remodeling of grafted bone mass were observed on serial radiographs from each patient. We noted the time required for the (1) appearance of new trabeculae bridging original iliac bone and grafted bone, (2) disappearance of the interface line between original iliac bone and grafted bone, and (3) appearance of new cortical bone in the lateral part of the grafted bone mass. On radiographs from the last followup, the percentage increase in projected horizontal coverage of the ilium just above the socket was calculated to estimate the ability of femoral head bulk autograft to restore acetabular bone stock. Radiographic findings of bone grafts at last followup were classified in three types: residual bone graft shadow (Fig. 4), partial sclerosis (Fig. 5), or complete incorporation (Fig. 6).

For patients who subsequently underwent revision during followup, clinical and radiographic data just before revision surgeries were used for analysis. Kaplan-Meier survival analysis [14] was used to calculate the probability of retention of the original prosthesis with $95 \%$ confidence intervals. The Kruskal-Wallis test was used to determine differences in coverage of the acetabular component by bone (CE, CE II, CE III), horizontal coverage above the
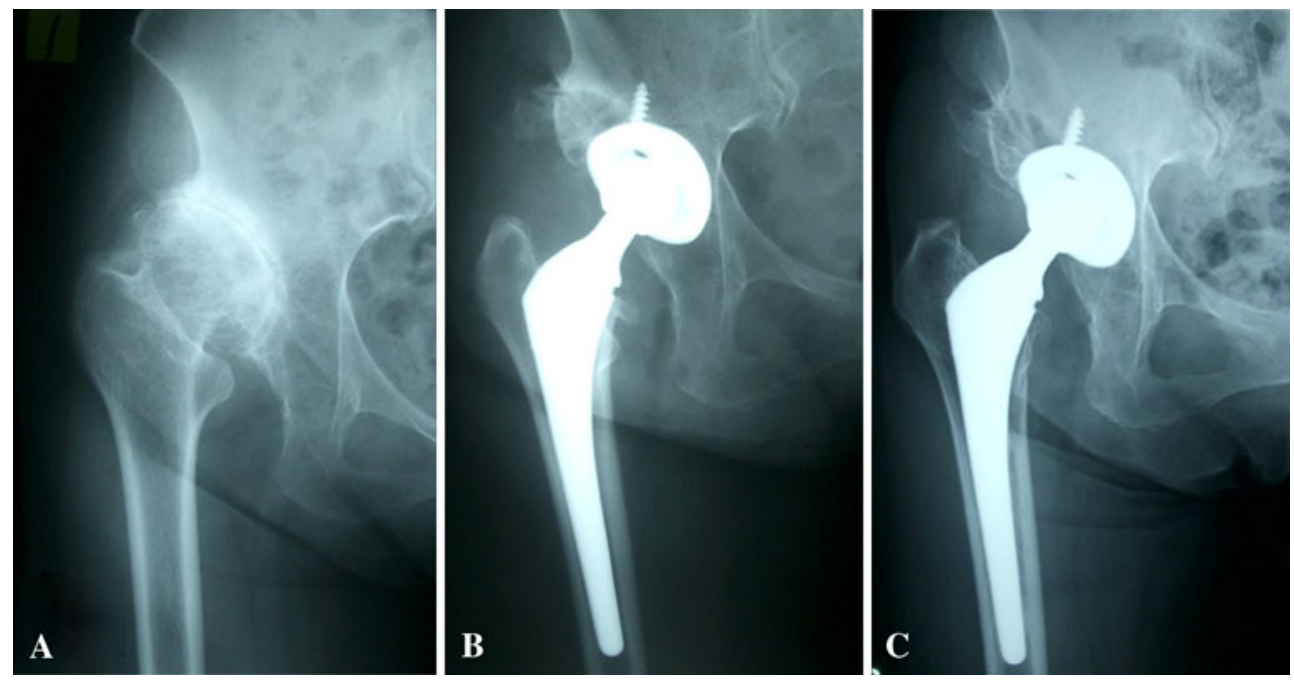

Fig. 4A-C The radiographs illustrate the case of a 63-year-old woman with Crowe Type III hip dysplasia. (A) A preoperative radiograph shows acetabular dysplasia. (B) A radiograph shows the hip 1 month after THA. (C) In a radiograph taken 10 years after THA, the graft bone shows good reorientation and the interface of host bone and graft bone is partially seen. 
Fig. 5A-C The radiographs illustrate the case of a 64-yearold woman with Crowe Type III hip dysplasia. (A) A preoperative radiograph shows acetabular dysplasia. (B) A radiograph shows the hip 1 month after THA. (C) In a radiograph taken 11 years after THA, the graft bone shows good reorientation but was partially sclerotic.
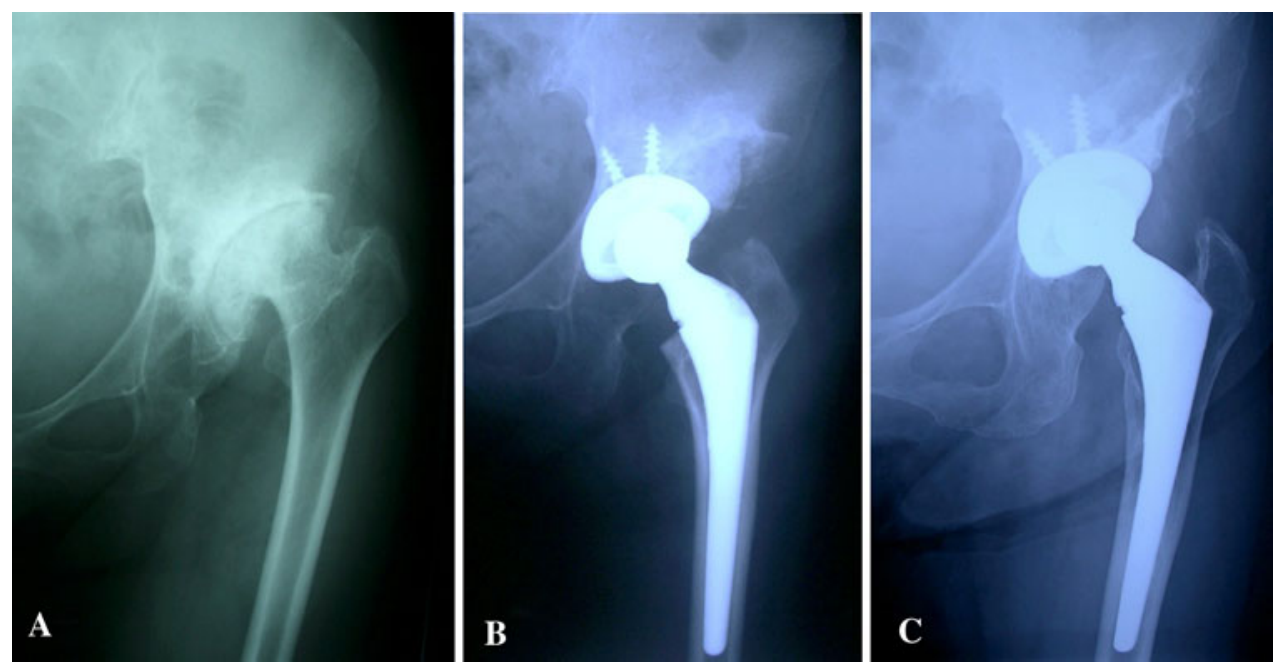

Fig. 6A-E The radiographs illustrate the case of a 51-yearold woman with Crowe Type II hip dysplasia. (A) A preoperative radiograph shows acetabular dysplasia. (B) A radiograph taken 3 months postoperatively shows bridging trabeculation. (C) A radiograph taken 12 months after THA shows graft remodeling. (D) A radiograph taken 1.6 years postoperatively shows trabecular reorientation. (E) On the last visit, graft bone volume was unchanged and the interface of the host bone was obscure.
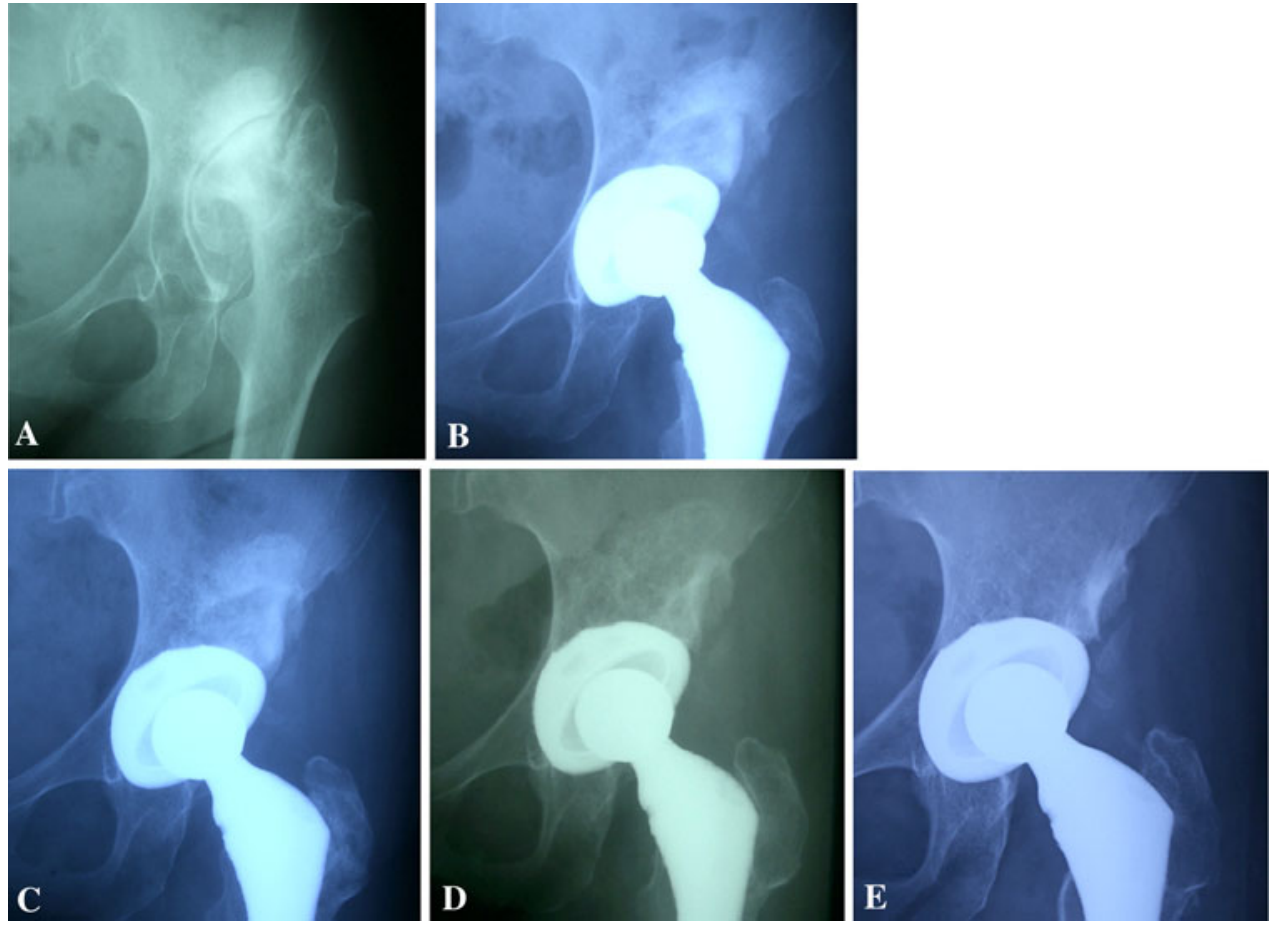

socket, and bone graft staging as defined by Knight et al. [15] to dislocation type as defined by Crowe et al. [4]. We used StatView ${ }^{\circledR}$ software (SAS Institute Inc, Cary, NC) for all analyses.

\section{Results}

The 10-year survival rate without acetabular revision for any reason (wear, osteolysis, loosening) was 94\% (95\% confidence interval, 92\%-96\%). The 10-year survival rate with loosening was $97 \%$ (95\% confidence interval,
95\%-99\%). Although we observed no patients with socket loosening attributable to collapse of the autograft, we did revise five hips during followup. Four hip prostheses were revised at $6.5,7,8.1$, and 13 years after the index procedure owing to severe polyethylene wear and periprosthetic osteolysis. In all of these patients, the cup was firmly fixed to the acetabulum at the time of revision. One patient had revision surgery at 9 years because of acetabular loosening. No radiolucent lines were apparent at the graft-implant interface at the last followup in 65 of the 77 cups (84\%), but thin $(<1-\mathrm{mm})$ radiolucent lines at the socket-graft interface without socket loosening were seen in 12 hips. 
The mean rate of head penetration into the polyethylene liner was $0.10 \mathrm{~mm} /$ year (range, $0-0.38 \mathrm{~mm} /$ year). Pelvic osteolytic lesions were observed adjacent to the acetabular component in eight hips. Femoral osteolytic lesions without loosening were observed in five hips with high wear rates.

The mean percentage of horizontal coverage of the acetabular component by host bone was $45 \%$ (range, $23 \%-$

Table 2. Radiographic results

\begin{tabular}{ll}
\hline Parameter & Value \\
\hline $\begin{array}{l}\text { Socket inclination }\left({ }^{\circ}\right)^{*} \\
\text { Hip center }(\mathrm{mm})\end{array}$ & $44(32-60)$ \\
$\quad$ Horizontal* & $30.4(20-39)$ \\
Vertical* & $23.7(13-37)$ \\
Graft coverage & $45(23-66)$ \\
Horizontal coverage $(\%)^{*}$ & $-3.4(-20-15)$ \\
Center-edge angle $\left({ }^{\circ}\right)^{*}$ & $13(12 \%)$ \\
Radiolucent lines in DeLee and Charnley zones (number of hips) \\
I & $3(3 \%)$ \\
II & $7(8 \%)$ \\
III
\end{tabular}

All results are at final followup except graft coverage, which was immediately postoperatively; * values are expressed as means, with ranges in parentheses.
66\%) (Table 2). On the last radiographs, the horizontal coverage of the ilium just above the socket was increased $44 \%$ on average. Projected horizontal coverage was not different according to the dislocation types of Crowe et al. (Table 3).

Mean CE improved from $-3.4^{\circ}$ (range, $-20^{\circ}-20^{\circ}$ ) to $46^{\circ}$ (CE II; range, $32^{\circ}-60^{\circ}$ ) immediately after surgery (Table 2). Coverage of the acetabular component by bone was similar for the dislocation types of Crowe et al. (Table 4).

Bridging trabeculae across the graft-host interface were seen in all cases by a mean of 3 months postoperatively (range, 2-6 months). Disappearance of the acetabular bone graft interface line on the AP view was noted at a mean of 8 months postoperatively (range, 3-18 months). Definite trabecular reorientation was seen in 69 of the 77 hips (90\%) at a mean of 24 months (range, 9-36 months). Trabecular reorientation in grafted bone was apt to be delayed in hips classified as Type IV (Crowe et al.), probably owing to grafting of larger bone bulk in this type (Table 5). The size of the grafted bone mass reduced slightly during followup. Bone mass reduction was recognized within 8 months and the incorporated bone mass was maintained thereafter. On the last radiographs, new radiodense lateral bands were frequent (in 71 hips) (Fig. 7). These bands were present in the region of the original subchondral portion of the femoral head graft.

Table 3. Classification by Crowe et al. and horizontal coverage*

\begin{tabular}{lllll}
\hline Crowe et al. type & Number of hips & Socket size $(\mathrm{mm})$ & \multicolumn{2}{l}{ Horizontal coverage $(\%)^{\dagger}$} \\
\cline { 4 - 5 } & & & Immediately postoperatively & Final followup \\
\hline I & 10 & $46.8(46-48)$ & $59.2(41-74)$ & $99(94-100)$ \\
II & 45 & $46.8(46-48)$ & $54.7(34-69)$ & 100 \\
III & 19 & $46.1(46-48)$ & $55.4(35-69)$ & $98(89-100)$ \\
IV & 9 & $43.3(42-46)$ & $61.4(44-77)$ & 0.73 \\
p value & & 0.0001 & 0.12 &
\end{tabular}

* Coverage was determined as illustrated in Fig. $3 ;{ }^{\dagger}$ values are expressed as means, with ranges in parentheses.

Table 4. Classification by Crowe et al. and center-edge angle

\begin{tabular}{lllll}
\hline Crowe et al. type & Number of hips & CE $\left(^{\circ}\right)^{*}$ & CE II $\left(^{\circ}\right)^{*}$ & CE III $\left(^{\circ}\right)^{*}$ \\
\hline I & 10 & $-2.5(-20-15)$ & $48.9(42-63)$ & $46.3(42-63)$ \\
II & 45 & $-4.0(-20-10)$ & $50.1(35-65)$ & $49.0(35-65)$ \\
III & 19 & $-4.4(-25-10)$ & $51.3(40-60)$ & $48.9(40-60)$ \\
IV & 9 & $2.2(-15-20)$ & $48.3(35-60)$ & $46.7(35-60)$ \\
P value & & 0.37 & 0.88 & 0.82 \\
\hline
\end{tabular}

* Values are expressed as means, with ranges in parentheses; $\mathrm{CE}=$ center-edge angle (angle between the vertical line of the socket center and the original lateral edge of the acetabulum [which was also the medial edge of the graft bone] immediately postoperatively); CE II $=$ angle between the vertical line of the socket center and the lateral edge of the graft bone immediately postoperatively; CE III = angle between the vertical line of the socket center and the lateral edge of the graft bone at final followup. 
Table 5. Classification by Crowe et al. and the staging of Knight et al.

\begin{tabular}{lllll}
\hline Crowe et al. type & Number of hips & \multicolumn{2}{l}{ Knight et al. staging (months)* } \\
\cline { 2 - 4 } & & Trabecular bridging & Remodeling & $7(3-18)$ \\
I & 10 & $3(2-6)$ & $8(3-18)$ & $14(9.6-24)$ \\
II & 45 & $3(2-6)$ & $8(3-18)$ & $19(12-36)$ \\
III & 19 & $3(2-6)$ & $9(3-18)$ & $24(19.2-36)$ \\
IV & 9 & $3(2-6)$ & 0.52 & $<0.003$ \\
P value & & 0.81 & $12-36)$ \\
\hline
\end{tabular}

* Values are expressed as means, with ranges in parentheses.

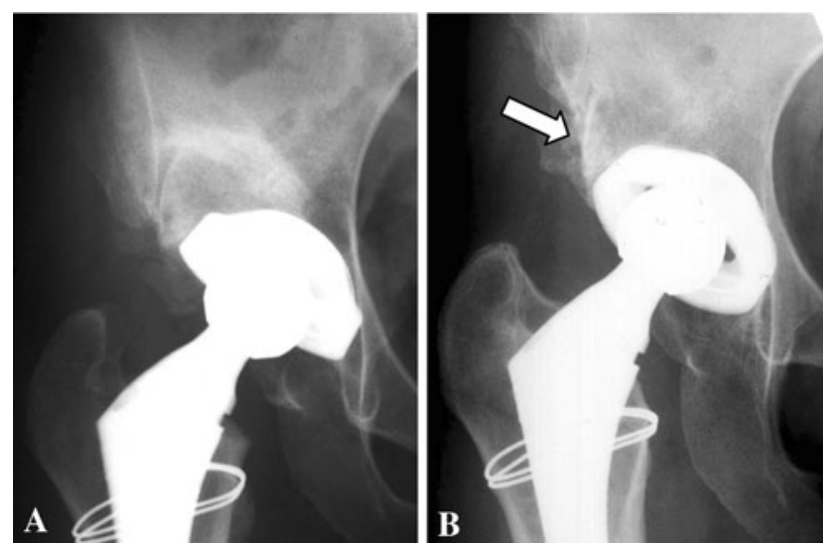

Fig. 7A-B The radiographs illustrate the case of a 33-year-old woman with Crowe Type II hip dysplasia. (A) A radiograph taken 3 months postoperatively shows bridging trabeculation. (B) A radiograph taken 3 years postoperatively shows trabecular reorientation. The arrow points to new radiodense lateral bands.

At the last followup, 60 of the 77 hips (78\%) were completely incorporated (Fig. 6E), six (8\%) had residual bone graft shadow (Fig. 4C), and 11 (14\%) had partial sclerosis (Fig. 5C).

Leg length was increased by $24.6 \mathrm{~mm}$ (range, 10$65 \mathrm{~mm}$ ) after surgery owing to the socket setting to the original acetabular cavity and distal shift of the femur.

The mean Merle d'Aubigné and Postel hip score improved from 9.1 (pain, 2.4; mobility, 3.4; function, 3.3) to 17.2 (pain, 5.9; mobility, 5.6; function, 5.7) after surgery. The mean horizontal hip center position from the interteardrop line was $30.4 \mathrm{~mm}$ (range, 20-39 mm) (Table 2). The mean height of the hip center was $23.7 \mathrm{~mm}$ (range, 13-37 mm) (Fig. 8). A high hip center was noted in six hips (7.8\%).

\section{Discussion}

In cases of acetabular dysplasia, deficient acetabula often provide inadequate coverage of the acetabular socket during THA. Bulk autogenous graft from the femoral head has

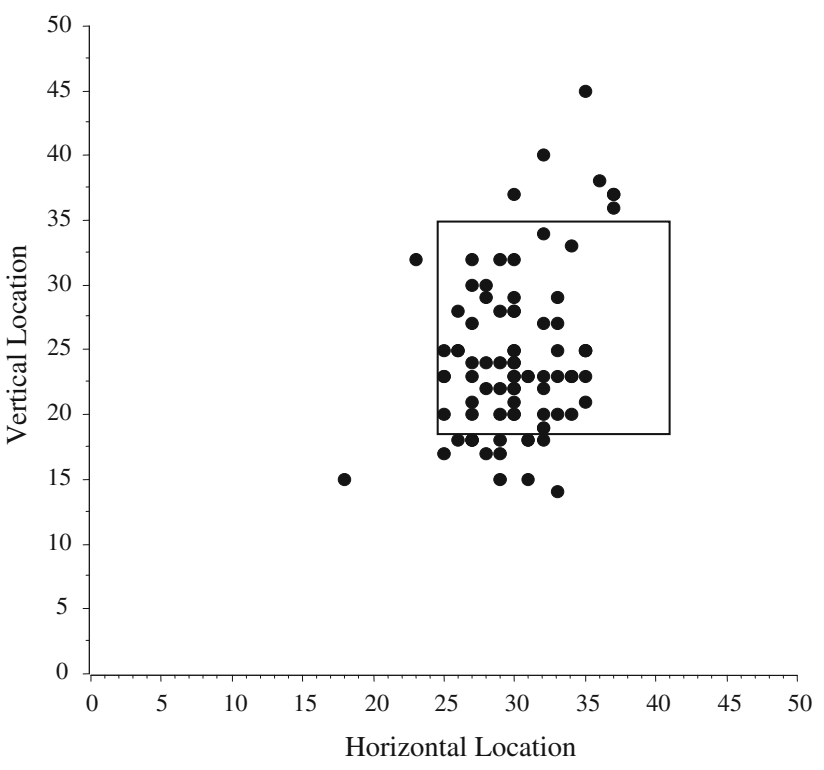

Fig. 8 A graph shows the postoperative center of the hip. The quadrangle indicates the range of hip center in a normal THA as described by Russotti and Harris [27].

been used to achieve better coverage, but the long-term benefits of this technique remain controversial. The major concerns with the surgical method of socket setting in the original acetabulum and augmentation with bone grafts to defective iliac bone are loosening of the socket caused by compromised mechanical strength of the graft during the process of incorporation and remodeling of graft bone and subsequent increases in the revision rate [7, 16, 22]. The use of bulk autograft thus remains controversial. We therefore determined (1) the 10-year survival rate with loosening, (2) coverage of the acetabular component by host bone, (3) chronologic radiographic changes in grafted bone during repair of acetabular bone deficiency in dysplastic hips, (4) leg lengthening achieved by the autografts, and (5) functional outcomes.

Our study has several limitations. First, we used only standard AP radiographs, reflecting 2-D images of the hips. As coverage of the socket by host and graft bone is $3-\mathrm{D}$, we 
could not capture the 3-D coverage (ie, AP). Second, we could not assess the rate or extent of invasion by reparative tissue into grafted bone and the metabolic condition of the bone in and around the autograft owing to a lack of data obtained by MRI, bone scintigraphy, and bone biopsy of grafts. However, during long-term followup, we observed reduced or absent radiodensity of the original floor of the pseudoacetabulum, reorientation of trabeculae in the graft, and generation of radiodense bands in the graft or along the outer surface of the graft original iliac outer wall to the lateral edge of the socket surface. Serial radiographs showed the appearance of lateral radiodense bands, representing a new outer cortical shell of the ilium and appearing to reflect a final event for successful incorporation of the bulk autograft; we observed no additional morphologic changes after the appearance of these lateral radiodense bands. Thus, there is considerable radiographic evidence of ultimate incorporation although we cannot establish the rate of incorporation. The lateral part of the acetabular roof plays an important role in load transfer. We thus presume large bone grafts have a functional role in load transfer. Third, we had only one observer for the radiographic images. Many of these measures (eg, repair, radiolucent lines, linear measures) are subject to interobserver variability and we have no data regarding the reliability of these measures in our patients. In particular, the data for repair times reflect only estimates. However, the observations regarding long-term repair would not likely be so subject to interobserver variability and these data would not affect the data for survival and revisions.

Based on their experience, Mulroy and Harris [22] advised against bulk autograft fabricated from the femoral head for dysplastic hips owing to a high rate of postoperative resorption and collapse of the bone graft. Based on these clinical experiences, some surgeons have recommended medial or high setting of the noncemented socket without bone grafting and have reported no clear loosening at an average of 10.6 years followup despite a medial or high shift of the anatomic hip center [13]. However, considering future potential loosening of the acetabular sockets in association with periprosthetic bone loss, revision surgery to restore hip function would become much more difficult in these patients owing to limited bone stock. To avoid such potential risks in revision THA, restoration of the acetabular bone defect in primary THA for the dysplastic hip in combination with socket setting in the original acetabulum to restore biomechanically favorable hip function would be desirable. Despite concerns regarding high rates of resorption and collapse, we observed no patient with graft collapse or progressive migration of the socket over 10 years' followup. Twelve of the 77 hips had periprosthetic radiolucent lines without graft collapse. We presume these were related to polyethylene wear from the conventional polyethylene liners and the use of microstructured sockets. These sockets may have experienced deformation of the liner with increased backside wear as described by Yamaguchi et al. [33]. We speculate the survival relates to the surgical techniques of bone grafting used in this series of cases. Tight fixation of the graft by these techniques would be particularly important in cases requiring a large graft where the mechanical load inevitably would be transmitted to the graft in the postoperative phase owing to increased socket coverage area by the grafted bone. With the improved porous surface, the socket adhered tightly to living host bone. Consequently, early mechanical load on the graft was reduced. Additional factors might contribute to satisfactory incorporation of grafts, but we could not elucidate these using radiographic data alone.

No clear consensus was reached regarding how much of the acetabular component can safely be covered by graft. Mulroy and Harris [22] found grafts covering less than $40 \%$ tended to collapse. As a result, they recommended at least $70 \%$ coverage of the acetabular component by host bone to give stability and allow adequate ingrowth on bone [22]. Initial socket stability should be achieved to the extent possible with host bone. Some authors believe surgeons should avoid dependence of achieving stability with greater than $60 \%$ of the grafted bone [26]. We found the mean percent coverage of the acetabular component by the bulk autograft was $45 \%$ (range, 23\%-66\%). We believe it is important to decrease the stress on the grafted bone. Although many of the bone grafts we used were relatively bulky, we obtained satisfactory results.

Iida et al. [11] reported delayed trabecular reorientation as a risk factor for socket loosening. They used a cemented socket with trabecular reorientation in $89 \%$ at an average of 32.5 months. The grafting method described by Knight et al. [15] also involved a cemented socket with either allograft or autograft, resulting in trabecular reorientation in $72 \%$ at an average of 16 months. Our grafting technique and cementless socket fixation achieved earlier trabecular reorientation at an average of 24 months.

Leg lengthening greater than $4 \mathrm{~cm}$ generally is accepted as a risk factor for nerve injury or palsy. Our THA method for DDH resulted in a mean postoperative lengthening of $24.6 \mathrm{~mm}$. No irreversible nerve damage was identified. In this study, we considered less than $4 \mathrm{~cm}$ a desirable level of lengthening for our patients with a mean height of $152 \mathrm{~cm}$.

The mean Merle d'Aubigné and Postel hip score improved from 9.1 preoperatively to 17.2 at last followup. Functional score improvements with medial and high settings of the noncemented socket in THA without bone grafting are similar [13]. Mallory et al. [18] reported frequent dislocation after THA in patients with DDH. The 
dislocation rate of $11 \%$ in the study by Ito et al. [13] with an acceptable high center was greater than our dislocation rate of $2.6 \%$. Silber and Engh [30] reported the potential for hip instability attributable to femoral-pelvic impingement was greater in patients with a high hip center. Intraoperative soft tissue tension was increased adequately by leg lengthening in our patients. An intraoperative check of the dislocation range showed very stable results, which might have been the reason for the low dislocation rate.

An uncemented THA augmented with a femoral head autograft offers a way to ensure anatomic placement of the socket in the AP projection if the THA and bone-grafting procedures are performed carefully. Repair of the deficient acetabulum by the autograft appeared to be complete within 3 years after surgery as judged radiographically. We speculate the high rate of graft incorporation and survival relates to key technical factors: (1) initial stability of the graft and cup; (2) impacted cancellous surfaces of the host and graft to facilitate long-term incorporation and remodeling; and (3) a porous surface of the largest possible sockets to ensure bone ingrowth.

Acknowledgments We thank Kunio Takaoka MD, for his valued contributions to this study.

Open Access This article is distributed under the terms of the Creative Commons Attribution Noncommercial License which permits any noncommercial use, distribution, and reproduction in any medium, provided the original author(s) and source are credited.

\section{References}

1. Anderson MJ, Harris WH. Total hip arthroplasty with insertion of the acetabular component without cement in hips with total congenital dislocation or marked congenital dysplasia. $J$ Bone Joint Surg Am. 1999;81:347-354.

2. Barrack RL, Newland CC. Uncemented total hip arthroplasty with superior acetabular deficiency: femoral head autograft technique and early clinical results. J Arthroplasty. 1990;5:159-167.

3. Bostman OM. Osteolytic changes accompanying degradation of absorbable fracture fixation implants. J Bone Joint Surg Br. 1991;73:679-682.

4. Crowe JF, Mani VJ, Ranawat CS. Total hip replacement in congenital dislocation and dysplasia of the hip. J Bone Joint Surg Am. 1979;61:15-23.

5. DeLee JG, Charnley J. Radiological demarcation of cemented sockets in total hip replacement. Clin Orthop Relat Res. 1976;121:20-32.

6. Garvin KL, Bowen MK, Salvati EA, Ranawat CS. Long-term results of total hip arthroplasty in congenital dislocation and dysplasia of hip: a follow-up note. J Bone Joint Surg Am. 1991;73:1348-1354.

7. Gerber SD, Harris TW. Femoral head autografting to augment acetabular deficiency in patients requiring total hip replacement: a minimum five-year and an average seven-year follow-up study. J Bone Joint Surg Am. 1986;68:1241-1248.

8. Gross AE, Catre MG. The use of femoral head autograft shelf reconstruction and cemented acetabular components in the dysplastic hip. Clin Orthop Relat Res. 1994;298:60-66.
9. Hendrich C, Mehling I. Cementless acetabular reconstruction and structural bone-grafting in dysplastic hips. J Bone Joint Surg Am. 2006;88:387-394

10. Hintermann B, Morscher EW. Total hip replacement with solid autologous femoral head graft for hip dysplasia. Arch Orthop Trauma Surg. 1995;114:137-144.

11. Iida $H$, Matsusue $Y$, Kawanabe K,Okumura H, Yamamuro T, Nakamura T. Cemented total hip arthroplasty with acetabular bone graft for developmental dysplasia. Long-term results and survivorship analysis. J Bone Joint Surg Br. 2000;82:176184.

12. Inao $\mathrm{S}$, Gotoh $\mathrm{E}$, Ando M. Total hip replacement using femoral neck bone to graft the dysplastic acetabulum: follow-up study of 18 patients with old congenital dislocation of the hip. $J$ Bone Joint Surg Br. 1994;76:735-739.

13. Ito $\mathrm{H}$, Matsuno $\mathrm{T}$, Minami $\mathrm{A}$, Aoki $\mathrm{Y}$. Intermediate-term results after hybrid total hip arthroplasty for the treatment of dysplastic hips. J Bone Joint Surg Am. 2003;85:1725-1732.

14. Kaplan EL, Meier P. Nonparametric estimation from incomplete observations. J Am Stat Assoc. 1958;53:457-481.

15. Knight JL, Fujii K, Atwater R, Grothaus L. Bone-grafting for acetabular deficiency during primary and revision total hip arthroplasty: a radiographic and clinical analysis. J Arthroplasty. 1993;8:371-382.

16. Lee BP, Cabanela ME, Wallrichs SL, Ilstrup DM. Bone-graft augmentation for acetabular deficiencies in total hip arthroplasty: results of long-term follow-up evaluation. J Arthroplasty. 1997; 12:503-510.

17. Livermore J, Ilstrup D, Morrey B. Effect of femoral head size on wear of polyethylene acetabular component. J Bone Joint Surg Am. 1990;72:518-528.

18. Mallory TH, Lombardi AV Jr, Fada RA, Herrington SM, Eberle RW. Dislocation after total hip arthroplasty using the anterolateral abductor split approach. Clin Orthop Relat Res. 1999;358:166-172.

19. Mcqueary FG, Johnston RC. Coxarthrosis after congenital dysplasia: treatment by total hip arthroplasty without acetabular bone grafting. J Bone Joint Surg Am. 1988;70:1140-1144.

20. Merle d'Aubigné R, Postel M. Functional results of hip arthroplasty with acrylic prosthesis. J Bone Joint Surg Am. 1954;36:451-475.

21. Morsi E, Gabuz D, Gross AE. Total hip arthroplasty with shelf grafts using uncemented cups: a long term follow up study. J Arthroplasty. 1996;11:81-85.

22. Mulroy RD Jr, Harris WH. Failure of acetabular autogenous grafts in total hip arthroplasty. Increasing incidence: a follow-up note. J Bone Joint Surg Am. 1990;72:1536-1540.

23. Pagnano W, Hanssen AD, Lewallen DG, Shaughnessy WJ. The effect of superior placement of the acetabular component on the rate of loosening after total hip arthroplasty. J Bone Joint Surg Am. 1996;78:1004-1014.

24. Raut VV, Stone MH, Siney PD, Wroblewski BM. Bulk autograft for a deficient acetabulum in Charnley low-friction arthroplasty: a 2-9 year follow-up study. J Arthroplasty. 1994;9: 393-398.

25. Ritter MA, Transik TM. Lateral acetabular bone graft in total hip arthroplasty: a three-to eight-year follow-up study without internal fixation. Clin Orthop Relat Res. 1985;193:156-159.

26. Rodriguez JA, Huk OL, Pellicci PM, Wilson PD Jr. Autogenous bone grafts from the femoral head for the treatment of acetabular deficiency in primary total hip arthroplasty with cement: long-term results. J Bone Joint Surg Am. 1995;77: $1227-1233$.

27. Russotti GM, Harris WH. Proximal placement of acetabular component in total hip arthroplasty: a long term follow-up study. J Bone Joint Surg Am. 1991;73:587-592. 
28. Shetty AA, Sharma P, Singh S, Tindall A, Kumar SV, Rand C. Bulk femoral-head autografting in uncemented total hip arthroplasty for acetabular dysplasia: results at 8 to 11 years follow-up. J Arthroplasty. 2004;19:706-713.

29. Shinar AA, Harris WH. Bulk structural autogenous grafts and allografts for reconstruction of the acetabulum in total hip arthroplasty: sixteen-year average follow-up. J Bone Joint Surg Am. 1997;79:159-168.

30. Silber DA, Engh CA. Cementless total hip arthroplasty with femoral head bone grafting for hip dysplasia. J Arthroplasty. 1990;5:231-240.
31. Spangehl MJ, Berry DJ, Trousdale RT, Cabanela ME. Uncemented acetabular components with bulk femoral head autograft for acetabular reconstruction in developmental dysplasia of the hip: results at five to twelve years. J Bone Joint Surg Am. 2001;83:1484-1489.

32. Sugano N, Nishii T, Nakata K, Masuhara K, Takaoka K. Polyethylene sockets and alumina ceramic heads in cemented total hip arthroplasty: a ten-year study. J Bone Joint Surg Br. 1995;77:548-556.

33. Yamaguchi M, Bauer TW, Hashimoto Y. Deformation of the acetabular polyethylene liner and the back side gap. J Arthroplasty. 1999;14:464-469. 